\title{
Structural and Electrochemical Characterizations of NiCo-S@GO composite as Supercapacitor Electrode
}

Jianhua $\mathrm{Yu}^{1}$, Xiancai Pang ${ }^{1}$, Xiaolei Gao ${ }^{2}$, Zhenxing Cui ${ }^{1}$, Qian Zhang ${ }^{1}$, Lina Sui ${ }^{1}$ and Lifeng Dong ${ }^{3}$

${ }^{1}$ Qingdao University of Science and Technology, Qingdao, Shandong, China (People's Republic),

${ }^{2}$ Shandong Sinocera Functional Material Co., Ltd, Dongying, Shandong, China (People's Republic),

${ }^{3}$ Hamline University, St. Paul, Minnesota, United States

Recently, binary transition metal sulfide has attracted special attention as electrode material for supercapacitors owing to high conductivity, excellent redox reversibility and super specific capacitance [1-2]. However, its practical applications are seriously hindered by its poor rate capability and cycling performance. Thus, it is imperative to develop high performance alternative candidate with high energy density and good cyclic stability for supercapacitors. Herein, graphene oxide (GO) was introduced in the synthesis of NiCo-MOF-74 (Ni/Co molar ratio =2:3), and its derived sulfide (NiCo-S @ GO) demonstrates superior electrochemical performance compared with NiCo-S.

In our experiments, $\mathrm{NiCo}-\mathrm{MOF}-74 @ \mathrm{GO}$ precursor was prepared by adding $\mathrm{GO}$ in the synthesis of $\mathrm{NiCo}-$ MOF-74, and the precursor was oxidized and sulfurized by heat treatment and solvothermal process successively to obtain NiCo-S@GO. For comparison, NiCo-S was prepared under the same conditions without GO. The morphology, structure and electrochemical properties of the samples were characterized by transmission electron microscopy (TEM, JEOL, JEM-2100 Thermo), X-ray diffraction (XRD, Rigaku, D-max- $\gamma \mathrm{A}$ ) analysis, and electrochemical workstation (CHI 760E), respectively.

NiCo-sulfide with rod structures is observed (Figure 1a), but there are abundant nanoparticles with about $10 \mathrm{~nm}$ dispersed on GO to form rod like shape (Figure 1b) or along the NiCo-sulfide rod (Figure 1c), suggesting that the addition of GO promotes the dispersion of crystal nucleus, but hinders the formation of rod-like structures. HRTEM images (Figure 1d-e) display well-resolved lattice fringes with interplanar distances of $0.16,0.18,0.24,0.28$, and $0.19 \mathrm{~nm}$ assigned to (440), (511), (400), (311), and (422) planes of $\mathrm{NiCo}_{2} \mathrm{~S}_{4}$, respectively, consistent with the XRD analysis (Figure 1f). The specific capacitances at various current densities obtained from galvanostatic charge-discharge (GCD) curves (Inset of Figure 2a) are shown in Figure 2a. It is observed that the specific capacitance of NiCo-S@GO is $1500 \mathrm{~F} \mathrm{~g}^{-1}$ lower than that of NiCo-S at $1 \mathrm{~A} \mathrm{~g}^{-1}$, while $791 \mathrm{~F} \mathrm{~g}^{-1}$ and $673 \mathrm{~F} \mathrm{~g}^{-1}$ at $50 \mathrm{~A} \mathrm{~g}^{-1}$ are retained for NiCo-S@GO and $\mathrm{NiCo}-\mathrm{S}$ with a rate capability of $53 \%$ and $35 \%$, respectively. Moreover, GCD measurements at $20 \mathrm{~A} \mathrm{~g}^{-1}$ for 1000 cycles were conducted to evaluate long-term cycling performance, and the specific capacitance retention of NiCo-S@GO can reach 80\%, higher than that of NiCo-S (77\%). The Nyquist plots (Inset of Figure $2 b)$ display that the equivalent series resistance $\left(\mathrm{R}_{\mathrm{S}}\right)$ of both samples are similar, while the chargetransfer resistance ( $R_{c t}$, obtained from the semicircle diameters in the intermediate frequency region) of $\mathrm{NiCo}-\mathrm{S} @ \mathrm{GO}$ is smaller than that of $\mathrm{NiCo}-\mathrm{S}$, indicating that the addition of $\mathrm{GO}$ enhances charge transportation which is benefit for increasing specific capacitance at higher current densities. While poor specific capacitance at low current density for NiCo-S@GO could be due to the decreased formation of rod-like NiCo-sulfide. This suggests that the addition of GO can improve the rate capability by reducing $\mathrm{R}_{\mathrm{ct}}$ and enhance the cycling stability of NiCo-S [3]. 

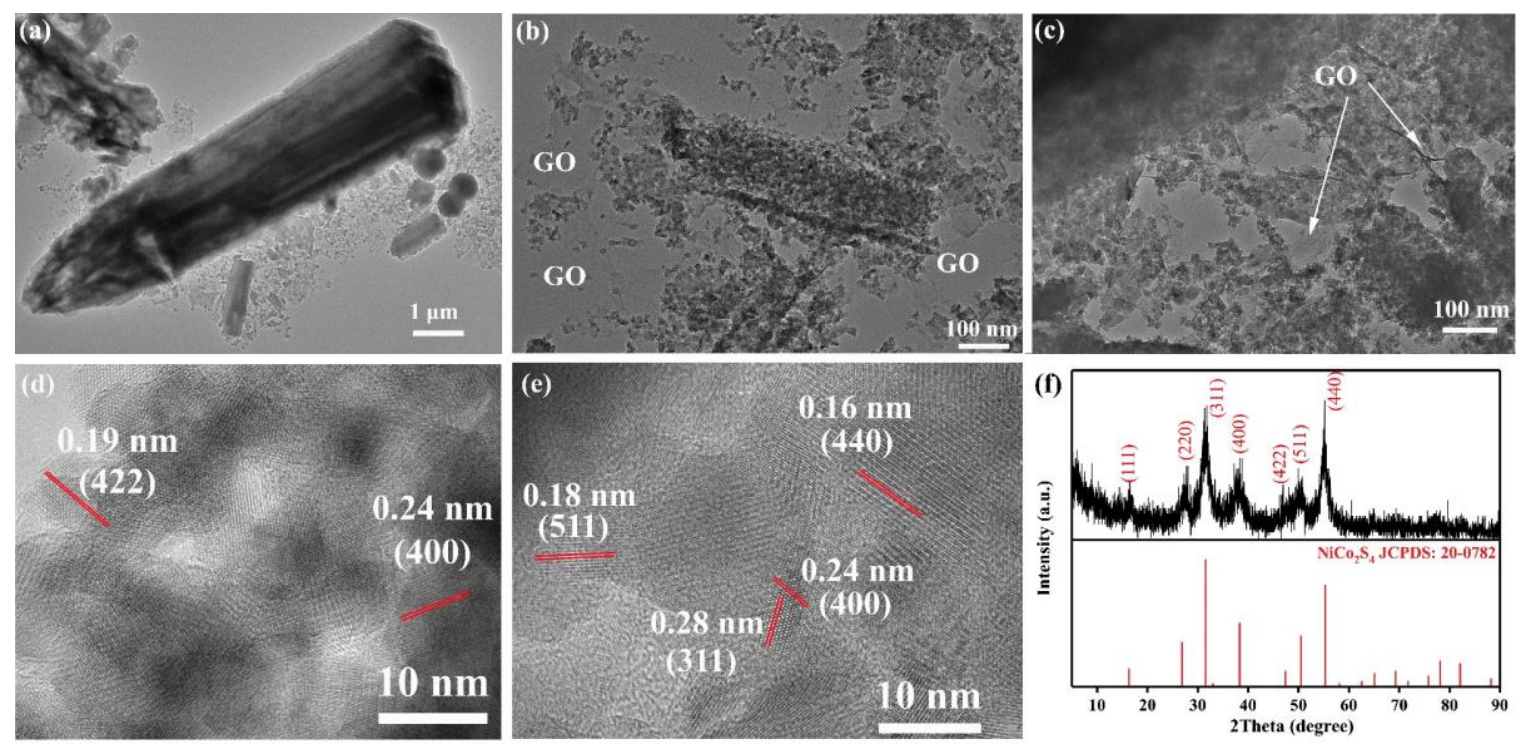

Figure 1. TEM images (a-c), HRTEM images (d, e) and XRD pattern (f) of NiCo-S@GO.
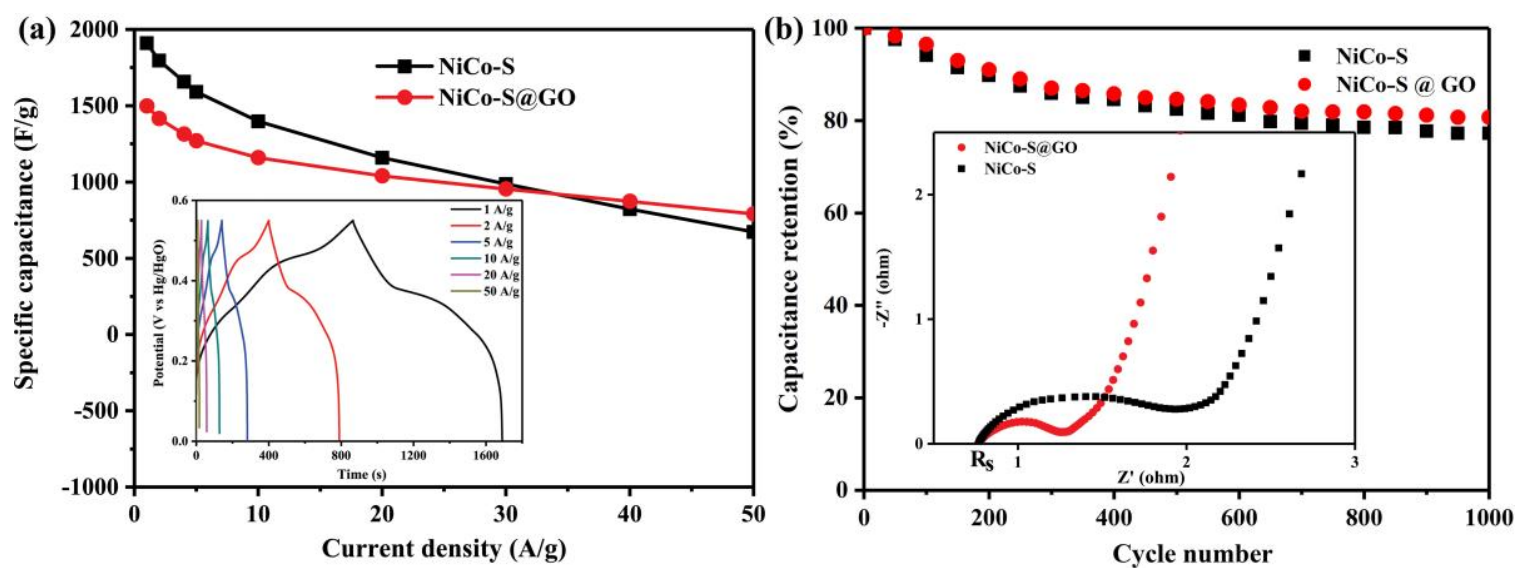

Figure 2. (a) Specific capacitance values at different current densities (inset is the GCD curves of the NiCo-S@GO) and (b) long-term cycling performance of NiCo-S and NiCo-S@GO (inset is the Nyquist plots).

\section{References}

[1] J. Yu et al, Energy Technol 7 (2019), p. 1900018.

[2] W. Lu, et al., Chemical Engineering Journal 382 (2019), p. 122943.

[3] This work was partially supported by the National Natural Science Foundation of China (51472174, 61604086, and 21776147), the International Science \& Technology Cooperation Program of China (2014DFA60150), the Department of Science and Technology of Shandong Province (ZR2018BB066 and 2016GGX104010), and the Department of Education of Shandong Province (J16LA14). L. F. Dong also thanks financial support from the Malmstrom Endowment Fund at Hamline University. 